\title{
POLARIZED LINE PROFILES IN PLANETARY NEBULAE
}

\author{
J.R.WALSH ${ }^{1}$ and R.E.S.CLEGG ${ }^{2}$ \\ ${ }^{1}$ Space Telescope Coordinating Facility, ESO, Karl-Schwarzchild-Strasse 2 \\ D-8046, Garching bei München, Germany \\ ${ }^{2}$ Royal Greenwich Observatory, Madingley Road, Cambridge, CB3 OHZ, United Kingdom
}

There is much direct and indirect evidence for the presence of dust in Planetary Nebulae $(\mathrm{PN})$ : variations in extinction across the face of the nebulae; IR emission with strong 25 and $60 \mu \mathrm{m}$ fluxes; broad near-IR emission lines of Silicate, SiC and PAH grains; optically thick lines, such as [C IV] $1550 \AA$, have lower strength on account of the increased path length due to dust scattering; a centro-symmetric pattern of polarization vectors in a few PN (Leroy et al., A\&A, 160, 171, 1986). An observational programme has begun to study the polarization profiles of bright emission lines in $\mathrm{PN}$ arising from dust scattering within the nebulae. The first results are discussed.

Six PN were observed with the $4.2 \mathrm{~m}$ William Herschel Telescope and the ISIS spectrograph. A Wave Plate Polarimeter with a half-wave plate located above the spectrograph slit was employed to measure linear polarization at high spectral resolution. Of the six PN observed, five (NGC 650, NGC 1535, NGC 2346, NGC 7027 and IC418) showed observable polarization in the emission lines and only one, NGC 2392, showed none detectable. In the core regions (generally the bright centre of the nebulae) the polarization is low or zero, accountable by interstellar polarization between the PN and the Earth. In 3 out of the 5 PN with detectable polarization, it is larger in the red wing of the emission line than in the blue wing. The offset regions observed in NGC 7027 (over the neutral and molecular halo) have very large polarization, upto $35 \%$ at [O III] $5007 \AA$.

A PN has strong line emission so it is expected that this would mask any polarization. However dust mixed with the gas scatters photons and since the relative velocity between two atoms in an expanding nebula is equivalent to a redshift, scattering occurs predominantly in the red wing of the line. Since the ernission is lower in the line wings, dilution of the polarized emission is less than in the line centre and the characteristic polarization profile can be seen. Some PN also show increased polarization in the blue wing indicating either that some of the dust is not well mixed with the ionized gas or does not simply partake in the expansion with the gas. The high polarization values in the outer halo of NGC 7027 can be explained in an identical way, however there is almost no local unpolarized emission to dilute the polarized flux and in addition scattering angles close to $90^{\circ}$ can occur.

A polarization model was constructed for NGC 7027 using a 3-D form of the nebula for the emitting gas and scattering by graphite dust. Apertures placed on the model nebula at positions corresponding to those observed could show both the effect of increased polarization in the red wing for line profiles at the centre of the nebula and the strong polarization profiles at offset positions over the neutral halo. Thus modelling the polarization profiles can provide useful information on the scattering properties of the grains (complementing the emission properties from IR data) and the extent and velocity field of the dust within PN. 